\title{
Dynamical Yang-Baxter Maps with an Invariance Condition
}

\author{
By
}

\author{
Youichi SHIBUKAWA*
}

\begin{abstract}
By means of left quasigroups $L=(L, \cdot)$ and ternary systems, we construct dynamical Yang-Baxter maps associated with $L, L$, and $(\cdot)$ satisfying an invariance condition that the binary operation $(\cdot)$ of the left quasigroup $L$ defines. Conversely, this construction characterize such dynamical Yang-Baxter maps. The unitary condition of the dynamical Yang-Baxter map is discussed. Moreover, we establish a correspondence between two dynamical Yang-Baxter maps constructed in this paper. This correspondence produces a version of the vertex-IRF correspondence.
\end{abstract}

\section{$\S 1$. Introduction}

Much attention has been directed to the quantum dynamical Yang-Baxter equation (QDYBE), a generalization of the quantum Yang-Baxter equation (QYBE) (for example, see [4]). The dynamical Yang-Baxter map (dynamical YB map) [13] is a set-theoretical solution to a version of the QDYBE.

Let $H$ and $X$ be nonempty sets, and $\phi$ a map from $H \times X$ to $H$. A map $R(\lambda): X \times X \rightarrow X \times X(\lambda \in H)$ is a dynamical $Y B$ map associated with $H, X$, and $\phi$, iff, for every $\lambda \in H, R(\lambda)$ satisfies the following equation on $X \times X \times X$.

$$
\text { (1.1) } R_{23}(\lambda) R_{13}\left(\phi\left(\lambda, X^{(2)}\right)\right) R_{12}(\lambda)=R_{12}\left(\phi\left(\lambda, X^{(3)}\right)\right) R_{13}(\lambda) R_{23}\left(\phi\left(\lambda, X^{(1)}\right)\right) .
$$

Communicated by M. Kashiwara. Received November 24, 2006. Revised April 19, 2007. 2000 Mathematics Subject Classification(s): Primary 81R50; Secondary 20F36, 20N05, $20 \mathrm{~N} 10$.

Key words: dynamical Yang-Baxter maps, unitary condition, vertex-IRF correspondence, (left) quasigroups, ternary systems, braid group relation.

This study was partially supported by the Ministry of Education, Science, Sports and Culture, Japan, grant-in-aid for Young Scientists (B), 15740001, 2005.

* Department of Mathematics, Faculty of Science, Hokkaido University, Sapporo 060-0810, Japan.

e-mail: shibu@math.sci.hokudai.ac.jp

(c) 2007 Research Institute for Mathematical Sciences, Kyoto University. All rights reserved. 
Here $R_{12}(\lambda), R_{12}\left(\phi\left(\lambda, X^{(3)}\right)\right), R_{23}\left(\phi\left(\lambda, X^{(1)}\right)\right)$, and others are the maps from $X \times X \times X$ to itself defined as follows: for $u, v, w \in X$,

$$
\begin{aligned}
& R_{12}(\lambda)(u, v, w)=(R(\lambda)(u, v), w) ; \\
& R_{12}\left(\phi\left(\lambda, X^{(3)}\right)\right)(u, v, w)=R_{12}(\phi(\lambda, w))(u, v, w) ; \\
& R_{23}\left(\phi\left(\lambda, X^{(1)}\right)\right)(u, v, w)=(u, R(\phi(\lambda, u))(v, w)) .
\end{aligned}
$$

If a map $R(\lambda)$ is a dynamical YB map associated with $H, X$, and $\phi$, then we denote it by $(R(\lambda) ; H, X, \phi)$. Two dynamical YB maps $\left(R^{(1)}\left(\lambda_{1}\right) ; H_{1}, X_{1}, \phi_{1}\right)$ and $\left(R^{(2)}\left(\lambda_{2}\right) ; H_{2}, X_{2}, \phi_{2}\right)$ are equal, iff

$$
H_{1}=H_{2}, X_{1}=X_{2}, \phi_{1}=\phi_{2}, \text { and } R^{(1)}(\lambda)=R^{(2)}(\lambda)
$$

for all $\lambda \in H_{1}\left(=H_{2}\right)$.

By the definition of the dynamical YB map, the Yang-Baxter map (YB map) [15], a set-theoretical solution to the QYBE $[2,16]$, is a dynamical YB map that is independent of the dynamical parameter $\lambda$. Geometric crystals [3], crystals [7], and semigroups of I-type [6] produce YB maps, and so do bijective 1-cocycles $[5,10]$. Let $A$ and $G$ be groups such that $A$ acts on $G$, and $\pi: A \rightarrow G$ a bijective 1-cocycle of the group $A$ with coefficients in the group $G$. This triplet $(A, G, \pi)$ gives birth to a bijective YB map [10, Theorems 1 and 2] with the invariance condition (4.6) (this invariance condition is called the compatibility condition in [10]).

By generalizing this method, dynamical YB maps were constructed in [13]. Let $L P=\left(L P, \cdot, e_{L P}\right)$ be a loop (see Definition 2.4), $G=\left(G, *, e_{G}\right)$ a group, and $\pi: L P \rightarrow G$ a set-theoretical bijection satisfying $\pi\left(e_{L P}\right)=$ $e_{G}$. Here $e_{L P}$ and $e_{G}$ are the unit elements of $L P$ and $G$, respectively. This triplet $(L P, G, \pi)$ produces a bijective dynamical YB map $R^{(G)}(\lambda)(2.6)$ with the invariance condition (2.5) (see below Theorem 2.5). We characterized this dynamical YB map (Theorem 2.5).

However, this characterization is inadequate; some dynamical YB maps with the invariance condition are not constructed in this way.

This paper clarifies a characterization of dynamical YB maps with the invariance condition. Let $L=(L, \cdot)$ be a left quasigroup (see Definition 2.1), $M=(M, \mu)$ a ternary system (Definition 3.1) satisfying (3.5) and (3.6), and $\pi$ a bijection from $L$ to $M$. The triplet $(L, M, \pi)$ produces a dynamical YB map $\left(R^{(L, M, \pi)}(\lambda) ; L, L,(\cdot)\right)(3.3)$ (Theorem 3.2). This dynamical YB map satisfies the invariance condition (3.4) that the binary operation $(\cdot)$ of the left quasigroup $L$ defines. This construction gives a characterization of the dynamical YB maps with the invariance condition (4.5) (Theorem 4.7). If the binary operation (.) 
of $L$ is associative, then every YB map on $L \times L$ with the invariance condition (4.6) is produced by a ternary system satisfying (3.5) and (3.6) (see Remark 4.8).

The organization of this paper is as follows. After summarizing the results of the work [13] in Section 2, we construct the dynamical YB map $R^{(L, M, \pi)}(\lambda)$ (3.3) with the invariance condition in Section 3 (Theorem 3.2). This dynamical YB map $R^{(L, M, \pi)}(\lambda)$ is a generalization of the YB map in [10] and the dynamical YB map in [13] (see Remarks 2.6 and 6.7). The dynamical YB map $R^{(L, M, \pi)}(\lambda)$ and the corresponding dynamical braiding map $\sigma^{(L, M, \pi)}(\lambda)(3.11)$ are expressed by means of the maps $s(a)(3.7)$ and $s$ (3.8) (see Lemma 3.5 and (7.3)), which satisfy the braid group relation (3.9). This braid group relation and Lemma 3.5 simplify the proof that the YB maps and the dynamical YB maps in $[10,13]$ satisfy $(1.1)$.

By means of categories $\mathcal{A}$ and $\mathcal{D}$ (Propositions 4.3 and 4.6), we characterize the dynamical YB maps associated with $L, L$, and $(\cdot)$ satisfying the invariance condition (4.5) in Sections 4 and 5 (Theorem 4.7).

Section 6 describes several examples of the ternary systems satisfying (3.5) and (3.6). For each $M=\left(G, \mu_{1}^{G}\right)(6.2),\left(G, \mu_{2}^{G}\right)(6.3),\left(G, \mu_{3}^{G}\right)(6.7)$, we give a characterization of the dynamical YB maps $R^{(L, M, \pi)}(\lambda)$.

Sections 7 and 8 deal with properties of the dynamical YB map $R^{(L, M, \pi)}(\lambda)$.

Let $M$ be a ternary system constructed in Section 6 . In Section 7 , we give a necessary and sufficient condition for the dynamical YB map $R^{(L, M, \pi)}(\lambda)$ to satisfy the unitary condition (2.8) (see Propositions 7.1, 7.3, and 7.5). Eq. (7.3) explains the reason that only the property (7.1) of the ternary system $M$ is needed in order for the dynamical YB map $R^{(L, M, \pi)}(\lambda)$ to satisfy the unitary condition (Theorem 2.7).

Section 8 gives a correspondence between two dynamical YB maps called an IRF-IRF correspondence (Proposition 8.1); furthermore, a vertex-IRF correspondence (8.1) is discussed. Eq. (7.3) induces this IRF-IRF correspondence. A motivation for producing these correspondences is the exchange matrix construction of the dynamical R-matrix by means of the fusion matrix (see Remark 8.2 ).

To end Introduction, the author would like to thank Professor Yas-Hiro Quano for advising him to investigate the vertex-IRF correspondence. 
Table 1. Multiplication table of $(\{1,2,3\}, *)$

\begin{tabular}{|c||c|c|c|}
\hline$*$ & 1 & 2 & 3 \\
\hline \hline 1 & 1 & 3 & 2 \\
\hline 2 & 2 & 1 & 3 \\
\hline 3 & 3 & 2 & 1 \\
\hline
\end{tabular}

\section{$\S 2 . \quad$ Background Information}

In this section, we briefly summarize the results of the work [13] after introducing definitions and notations used in this work.

Definition 2.1. $\quad(L, \cdot)$ is said to be a left quasigroup, iff $L$ is a non-empty set, together with a binary operation $(\cdot)$ having the property that, for all $u$, $w \in L$, there uniquely exists $v \in L$ such that $u \cdot v=w$ (cf. right quasigroups in $[14$, Section I.4.3]).

By this definition, the left quasigroup $(L, \cdot)$ has another binary operation $\backslash_{L}$ called the left division [14, Section I.2.2]; we denote by $u \backslash_{L} w$ the unique element $v \in L$ satisfying $u \cdot v=w$.

$$
u \backslash_{L} w=v \Leftrightarrow u \cdot v=w .
$$

Definition 2.2. A quasigroup $(Q, \cdot)$ is a left quasigroup satisfying that, for all $v, w \in Q$, there uniquely exists $u \in Q$ such that $u \cdot v=w$ (see [12, Definition I.1.1] and [14, Section I.2]).

The binary operation on a quasigroup is not always associative.

Example 2.3. We define the binary operation $(*)$ on the set $\{1,2,3\}$ of three elements by Table 1 . Here $1 * 2=3$. Then $(\{1,2,3\}, *)$ is a quasigroup, because each element in $\{1,2,3\}$ appears once and only once in each row and in each column of Table 1 [12, Theorem I.1.3]. This binary operation $(*)$ is not associative, since $(1 * 2) * 3 \neq 1 *(2 * 3)$.

Definition 2.4. A loop $\left(L P, \cdot, e_{L P}\right)$ is a quasigroup $(L P, \cdot)$ satisfying that there exists an element $e_{L P} \in L P$ such that $u \cdot e_{L P}=e_{L P} \cdot u=u$ for all $u \in L P$ [12, Definition I.1.10].

Because the above element $e_{L P} \in L P$ is uniquely determined, we call $e_{L P}$ the unit element of the loop $\left(L P, \cdot, e_{L P}\right)$. 
The group is a loop. To be more precise, the group is an associative quasigroup, and vice versa [12, Theorem I.1.7 and Definition I.1.9].

We shall simply denote by $L, Q$, and $L P$ a left quasigroup $(L, \cdot)$, a quasigroup $(Q, \cdot)$, and a loop $\left(L P, \cdot, e_{L P}\right)$, respectively; moreover, the symbol $u v$ will be used in place of $u \cdot v$.

Next task is to demonstrate the main theorem of [13]. Let $L P=(L P, \cdot$, $\left.e_{L P}\right)$ be a loop, $G=\left(G, *, e_{G}\right)$ a group, and $\pi: L P \rightarrow G$ a (set-theoretical) bijection satisfying $\pi\left(e_{L P}\right)=e_{G}$. For $u \in L P$, we define the map $\theta(u)$ from $G$ to itself by

$$
\theta(u)(x)=\pi(u)^{-1} * \pi\left(u \pi^{-1}(x)\right) \quad(x \in G) .
$$

Here $\pi(u)^{-1}$ is the inverse of the element $\pi(u)$ of the group $G$. This map $\theta(u)$ is bijective; $\theta(u)^{-1}(x)=\pi\left(u \backslash_{L P} \pi^{-1}(\pi(u) * x)\right)(x \in G)$.

Let $\xi_{\lambda}^{(G)}(u)$ and $\eta_{\lambda}^{(G)}(u)(\lambda, u \in L P)$ denote the following maps from $L P$ to itself: for $v \in L P$,

$$
\begin{aligned}
& \xi_{\lambda}^{(G)}(u)(v)=\pi^{-1} \theta(\lambda)^{-1} \theta(\lambda u) \pi(v) ; \\
& \eta_{\lambda}^{(G)}(u)(v)=\left(\lambda \xi_{\lambda}(v)(u)\right) \backslash_{L P}((\lambda v) u) .
\end{aligned}
$$

Theorem 3.7 in [13] implies Theorem 2.5.

Theorem 2.5. Let $\xi_{\lambda}(u)$ and $\eta_{\lambda}(v)(\lambda, u, v \in L P)$ be maps from LP to itself. The following conditions are equivalent:

(1) There exist a group $G=\left(G, *, e_{G}\right)$ and a bijection $\pi: L P \rightarrow G$ satisfying $\pi\left(e_{L P}\right)=e_{G}, \xi_{\lambda}(u)=\xi_{\lambda}^{(G)}(u)$, and $\eta_{\lambda}(v)=\eta_{\lambda}^{(G)}(v)$ for all $\lambda, u, v \in L P ;$

(2) The maps $\xi_{\lambda}(u)$ and $\eta_{\lambda}(v)$ satisfy the properties below.

$$
\begin{aligned}
& \xi_{\lambda}(u) \xi_{\lambda u}(v)=\xi_{\lambda}\left(\lambda \backslash_{L P}((\lambda u) v)\right) \quad(\forall \lambda, u, v \in L P), \\
& \eta_{\lambda \xi_{\lambda}(u)(v)}(w)\left(\eta_{\lambda}(v)(u)\right)=\eta_{\lambda}((\lambda u) \backslash L P \\
& \\
& \left.\left.\left(\lambda \xi_{\lambda}((u)(v)) \eta_{\lambda}(v)(u)=(\lambda u) v\right) w\right)\right)(u) \\
& \xi_{\lambda}\left(e_{L P}\right)=\eta_{\lambda}\left(e_{L P}\right)=\operatorname{id}_{L P} \quad(\forall \lambda, u, v, w \in L P), \\
& (\forall \lambda \in L P) .
\end{aligned}
$$

We define the map $R^{(G)}(\lambda)(\lambda \in L P)$ from $L P \times L P$ to itself by

$$
R^{(G)}(\lambda)(u, v)=\left(\eta_{\lambda}^{(G)}(v)(u), \xi_{\lambda}^{(G)}(u)(v)\right) \quad(u, v \in L P) .
$$

From Propositions 3.3 and 5.1 in [13] and the above theorem, this map $R^{(G)}(\lambda)$ is a bijective dynamical YB map associated with $L P, L P$, and $(\cdot)$. 
Remark 2.6. This method produces all YB maps constructed in the work [10]. We suppose that $L P$ is a group and that the map $\theta(u)$ satisfies

$$
\theta(u v)=\theta(u) \theta(v) \quad(\forall u, v \in L P) .
$$

The definition (2.2) of the map $\theta(u)$ and (2.7) immediately induce that $\pi$ is a bijective 1-cocycle of $L P$ with coefficients in $G$ [10, (8)]. Because of (2.3) and (2.7), the map $\xi_{\lambda}^{(G)}(u)(2.3)$ is independent of the dynamical parameter $\lambda$. Since $L P$ is a group, the map $\eta_{\lambda}^{(G)}(v)(2.4)$ is also independent of $\lambda$. By the definition of the YB map in [10, Case 2 in the proof of Theorem 2], the map $R^{(G)}(\lambda)(2.6)$ is the YB map in [10].

Next we shall show a necessary and sufficient condition for the dynamical YB map $R^{(G)}(\lambda)(2.6)$ to satisfy the unitary condition.

Let $R(\lambda)$ be a dynamical YB map associated with $H, X$, and $\phi$. This dynamical YB map $R(\lambda)$ is said to satisfy the unitary condition [13, Section 5], iff

$$
R(\lambda) P_{X} R(\lambda)=P_{X} \quad(\forall \lambda \in H) .
$$

Here we denote by $P_{X}$ the map from $X \times X$ to itself defined by

$$
P_{X}(u, v)=(v, u) \quad(u, v \in X) .
$$

Theorem 2.7 (Corollary 5.6 in [13]). The dynamical YB map $R^{(G)}(\lambda)$ (2.6) satisfies the unitary condition, if and only if the group $G$ is abelian.

Before ending this section, let us introduce dynamical braiding maps (see [13, Section 2]).

Definition 2.8. $\quad$ Let $H$ and $X$ be nonempty sets, and $\phi$ a map from $H \times X$ to $H$. A map $\sigma(\lambda): X \times X \rightarrow X \times X(\lambda \in H)$ is a dynamical braiding map associated with $H, X$, and $\phi$, iff, for every $\lambda \in H, \sigma(\lambda)$ satisfies the following equation on $X \times X \times X$.

$$
\sigma(\lambda)_{12} \sigma\left(\phi\left(\lambda, X^{(1)}\right)\right)_{23} \sigma(\lambda)_{12}=\sigma\left(\phi\left(\lambda, X^{(1)}\right)\right)_{23} \sigma(\lambda)_{12} \sigma\left(\phi\left(\lambda, X^{(1)}\right)\right)_{23} .
$$

The concepts of the dynamical braiding map and the dynamical YB map are exactly the same.

Proposition 2.9 (Proposition 2.1 in [13]). $\quad$ Let $R(\lambda)$ and $\sigma(\lambda)(\lambda \in H)$ be maps from $X \times X$ to itself satisfying $\sigma(\lambda)=P_{X} R(\lambda)$ for all $\lambda \in H$. Here $P_{X}$ is the map (2.9). The map $R(\lambda)$ is a dynamical $Y B$ map associated with $H$, $X$, and $\phi$, if and only if the map $\sigma(\lambda)$ is a dynamical braiding map associated with $H, X$, and $\phi$. 


\section{$\S 3 . \quad$ Construction}

Our main aim in the present section is to show how to construct dynamical YB maps. This is a generalization of the works [10, 13] (see Remarks 2.6 and $6.7)$.

Definition 3.1. A ternary system $(M, \mu)$ is a pair of a nonempty set $M$ and a ternary operation $\mu: M \times M \times M \rightarrow M$.

We shall simply denote by $M$ a ternary system $(M, \mu)$.

Let $L=(L, \cdot)$ be a left quasigroup, $M=(M, \mu)$ a ternary system, and $\pi: L \rightarrow M$ a (set-theoretical) bijection. For $\lambda, u \in L$, we define the maps $\xi_{\lambda}^{(L, M, \pi)}(u): L \rightarrow L$ and $\eta_{\lambda}^{(L, M, \pi)}(u): L \rightarrow L$ as follows: for $v \in L$,

$$
\begin{aligned}
& \xi_{\lambda}^{(L, M, \pi)}(u)(v)=\lambda \backslash_{L} \pi^{-1}(\mu(\pi(\lambda), \pi(\lambda u), \pi((\lambda u) v))) ; \\
& \eta_{\lambda}^{(L, M, \pi)}(u)(v)=\left(\lambda \xi_{\lambda}^{(L, M, \pi)}(v)(u)\right) \backslash_{L}((\lambda v) u) .
\end{aligned}
$$

Here $\backslash_{L}$ is the left division (2.1) of the left quasigroup $L$.

Let $R^{(L, M, \pi)}(\lambda)(\lambda \in L)$ denote the map from $L \times L$ to itself defined by

$$
R^{(L, M, \pi)}(\lambda)(u, v)=\left(\eta_{\lambda}^{(L, M, \pi)}(v)(u), \xi_{\lambda}^{(L, M, \pi)}(u)(v)\right) \quad(u, v \in L) .
$$

Since $L$ is a left quasigroup, (3.2) is equivalent to the following invariance condition of the map $R^{(L, M, \pi)}(\lambda)$ (see Remark 4.4).

$$
\left(\lambda \xi_{\lambda}^{(L, M, \pi)}(u)(v)\right) \eta_{\lambda}^{(L, M, \pi)}(v)(u)=(\lambda u) v \quad(\forall \lambda, u, v \in L) .
$$

Theorem 3.2. The map $R^{(L, M, \pi)}(\lambda)(3.3)$ is a dynamical YB map associated with $L, L$, and $(\cdot)$, if and only if the ternary system $M$ satisfies the following equations for all $a, b, c, d \in M$ :

$$
\begin{aligned}
& \mu(a, \mu(a, b, c), \mu(\mu(a, b, c), c, d))=\mu(a, b, \mu(b, c, d)) ; \\
& \mu(\mu(a, b, c), c, d)=\mu(\mu(a, b, \mu(b, c, d)), \mu(b, c, d), d) .
\end{aligned}
$$

This theorem induces that the triplet $(L, M, \pi)$ with (3.5) and (3.6) gives birth to a dynamical YB map $R^{(L, M, \pi)}(\lambda)(3.3)$ associated with $L, L$, and (·) satisfying the invariance condition (3.4).

Section 6 describes several ternary systems with (3.5) and (3.6).

Let $a$ be an element of the ternary system $M$. For the proof of Theorem 3.2, we need the maps $s(a): M \times M \rightarrow M \times M$ and $s: M \times M \times M \rightarrow M \times M \times M$ : for $x, y, z \in M$,

$$
\begin{aligned}
& s(a)(x, y)=(\mu(a, x, y), y) \\
& s(x, y, z)=(x, \mu(x, y, z), z) .
\end{aligned}
$$


Lemma 3.3. The maps $s(a)_{12}$ and $s$ satisfy the braid group relation

$$
s(a)_{12} s s(a)_{12}=s s(a)_{12} s \quad(\forall a \in M),
$$

if and only if the ternary system $M$ satisfies (3.5) and (3.6).

Proof. The proof is straightforward.

Let $\lambda$ be an element of the left quasigroup $L$, and let $f_{\lambda}$ denote the following map from $L \times L \times L$ to itself.

$$
f_{\lambda}(u, v, w)=(\lambda u,(\lambda u) v,((\lambda u) v) w) \quad(u, v, w \in L) .
$$

Lemma 3.4. The map $f_{\lambda}$ is bijective; $f_{\lambda}^{-1}(u, v, w)=\left(\lambda \backslash_{L} u, u \backslash_{L} v\right.$, $\left.v \backslash_{L} w\right)(u, v, w \in L)$.

We define the maps $\sigma^{(L, M, \pi)}(\lambda): L \times L \rightarrow L \times L$ and $\sigma^{(L, M, \pi)}\left(\lambda L^{(1)}\right)_{23}:$ $L \times L \times L \rightarrow L \times L \times L$ as follows: for $u, v, w \in L$,

$$
\begin{aligned}
& \sigma^{(L, M, \pi)}(\lambda)(u, v)=\left(\xi_{\lambda}^{(L, M, \pi)}(u)(v), \eta_{\lambda}^{(L, M, \pi)}(v)(u)\right) \\
& \sigma^{(L, M, \pi)}\left(\lambda L^{(1)}\right)_{23}(u, v, w)=\left(u, \sigma^{(L, M, \pi)}(\lambda u)(v, w)\right) .
\end{aligned}
$$

Lemma 3.5. The maps $\sigma^{(L, M, \pi)}(\lambda)_{12}$ and $\sigma^{(L, M, \pi)}\left(\lambda L^{(1)}\right)_{23}$ are expressed by means of the maps $s(\pi(\lambda))$ and $s$, respectively:

$$
\begin{aligned}
& \sigma^{(L, M, \pi)}(\lambda)_{12}=f_{\lambda}^{-1}\left(\pi^{-1} \times \pi^{-1} \times \pi^{-1}\right) s(\pi(\lambda))_{12}(\pi \times \pi \times \pi) f_{\lambda} \\
& \sigma^{(L, M, \pi)}\left(\lambda L^{(1)}\right)_{23}=f_{\lambda}^{-1}\left(\pi^{-1} \times \pi^{-1} \times \pi^{-1}\right) s(\pi \times \pi \times \pi) f_{\lambda} .
\end{aligned}
$$

Proof of Theorem 3.2. From Lemmas 3.3, 3.4, and 3.5, $\sigma^{(L, M, \pi)}(\lambda)$ is a dynamical braiding map associated with $L, L$, and (.) (see Definition 2.8), if and only if the ternary system $M$ satisfies (3.5) and (3.6).

Proposition 2.9, (3.3), and (3.11) complete the proof.

\section{$\S 4 . \quad$ Characterization}

This section clarifies a characterization of the dynamical YB map with the invariance condition (4.5); this map is exactly the dynamical YB map $R^{(L, M, \pi)}(\lambda)(3.3)$ constructed in the previous section.

To give a characterization, we need categories $\mathcal{A}$ and $\mathcal{D}$ (cf. [13, Section $3]$ ). For category theory, see $[8,11]$. Let $L=(L, \cdot)$ be a left quasigroup (see Definition 2.1), $M=(M, \mu)$ a ternary system (Definition 3.1) satisfying (3.5) 
and (3.6), and $\pi: L \rightarrow M$ a bijection. We denote by $L M B$ the set of all such triplets $(L, M, \pi)$.

Triplets $(L,(M, \mu), \pi)$ and $\left(L^{\prime},\left(M^{\prime}, \mu^{\prime}\right), \pi^{\prime}\right) \in L M B$ are equivalent, iff $L=$ $L^{\prime}$ as left quasigroups and the map $h:=\pi^{\prime} \pi^{-1}: M \rightarrow M^{\prime}$ is a homomorphism of ternary systems; that is, the map $h: M \rightarrow M^{\prime}$ satisfies

$$
h(\mu(a, b, c))=\mu^{\prime}(h(a), h(b), h(c)) \quad(\forall a, b, c \in M) .
$$

This is an equivalence relation, and we write it in the form $(L, M, \pi) \sim\left(L^{\prime}\right.$, $\left.M^{\prime}, \pi^{\prime}\right)$.

Let $[(L, M, \pi)]$ denote the equivalence class to which $(L, M, \pi) \in L M B$ belongs, $O b(\mathcal{A})$ the class of all equivalence classes with respect to this relation.

By the definition of the relation $\sim$, all the left quasigroups $L$ in representatives $(L, M, \pi)$ of $V \in O b(\mathcal{A})$ are the same. We denote by $L_{V}$ the left quasigroup $L$.

Definition 4.1. Let $V$ and $V^{\prime}$ be elements of $O b(\mathcal{A})$. We say that $f: V \rightarrow V^{\prime}$ is an element of $\operatorname{Hom}(\mathcal{A})$, iff $f: L_{V} \rightarrow L_{V^{\prime}}$ is a homomorphism of left quasigroups such that $\pi^{\prime} f \pi^{-1}: M \rightarrow M^{\prime}$ is a homomorphism (4.1) of ternary systems for all representatives $\left(L_{V}, M, \pi\right) \in V$ and $\left(L_{V^{\prime}}, M^{\prime}, \pi^{\prime}\right) \in V^{\prime}$.

Remark 4.2. On account of the definition of the equivalence relation $\sim$, $f: V \rightarrow V^{\prime} \in \operatorname{Hom}(\mathcal{A})$, iff $f: L_{V} \rightarrow L_{V^{\prime}}$ is a homomorphism of left quasigroups and there exist representatives $\left(L_{V}, M, \pi\right) \in V$ and $\left(L_{V^{\prime}}, M^{\prime}, \pi^{\prime}\right) \in V^{\prime}$ such that $\pi^{\prime} f \pi^{-1}: M \rightarrow M^{\prime}$ is a homomorphism of ternary systems.

Proposition 4.3. $\quad \mathcal{A}$ is a category: its objects are the elements of $\mathrm{Ob}(\mathcal{A})$; its morphisms are the elements of $\operatorname{Hom}(\mathcal{A})$; the identity id and the composition ○ of the category $\mathcal{A}$ are defined as follows:

$$
\begin{aligned}
& \text { for } V \in O b(\mathcal{A}), \operatorname{id}_{V}(u)=u\left(u \in L_{V}\right) \\
& \text { for } f: V \rightarrow V^{\prime}, g: V^{\prime} \rightarrow V^{\prime \prime} \in \operatorname{Hom}(\mathcal{A})\left(V, V^{\prime}, V^{\prime \prime} \in O b(\mathcal{A})\right), \\
& (g \circ f)(u)=g(f(u)) \quad\left(u \in L_{V}\right) .
\end{aligned}
$$

The next task is to introduce a category $\mathcal{D}$. Let $L=(L, \cdot)$ be a left quasigroup, and $R(\lambda)(\lambda \in L)$ a map from $L \times L$ to itself. We denote by $\xi_{\lambda}(u)$ and $\eta_{\lambda}(v)(\lambda, u, v \in L)$ the following maps from $L$ to $L$.

$$
\left(\eta_{\lambda}(v)(u), \xi_{\lambda}(u)(v)\right)=R(\lambda)(u, v) .
$$

Let us suppose that this map $R(\lambda)$ is a dynamical YB map associated with $L, L$, and $(\cdot)$ satisfying the invariance condition below:

$$
\left(\lambda \xi_{\lambda}(u)(v)\right) \eta_{\lambda}(v)(u)=(\lambda u) v \quad(\forall \lambda, u, v \in L) .
$$


Remark 4.4. To be more precise, Eq. (4.5) is the invariance condition for the corresponding dynamical braiding map $\sigma(\lambda)=P_{L} R(\lambda)$ (for the map $P_{L}$, see $\left.(2.9)\right)$.

We denote by $\operatorname{Ob}(\mathcal{D})$ the class of all such pairs $(L, R(\lambda))$.

Definition 4.5. $\quad$ Let $V=(L, R(\lambda))$ and $V^{\prime}=\left(L^{\prime}, R^{\prime}\left(\lambda^{\prime}\right)\right)$ be elements of $\operatorname{Ob}(\mathcal{D})$. We say that $f: V \rightarrow V^{\prime}$ is an element of $\operatorname{Hom}(\mathcal{D})$, iff $f: L \rightarrow L^{\prime}$ is a homomorphism of left quasigroups satisfying $R^{\prime}(f(\lambda))(f \times f)=(f \times f) R(\lambda)$ for all $\lambda \in L$.

Proposition 4.6. $\quad \mathcal{D}$ is a category: its objects are the elements of $O b(\mathcal{D})$; its morphisms are the elements of $\operatorname{Hom}(\mathcal{D})$; the definitions of the identity id and the composition $\circ$ are similar to (4.2) and (4.3).

Theorem 4.7 gives a characterization of the dynamical YB maps with the invariance condition (4.5).

Theorem 4.7. The category $\mathcal{A}$ is isomorphic to the category $\mathcal{D}$.

The next section will be devoted to the proof of this theorem; we shall explicitly construct functors $S: \mathcal{A} \rightarrow \mathcal{D}$ and $T: \mathcal{D} \rightarrow \mathcal{A}$ satisfying $T S=\mathrm{id}_{\mathcal{A}}$ and $S T=\operatorname{id}_{\mathcal{D}}$.

Remark 4.8. Theorem 4.7 produces an application of YB maps. Let $L$ be an associative left quasigroup and $R$ a YB map defined on the set $L \times L$. We denote by $\xi(u)$ and $\eta(v)(u, v \in L)$ the maps from $L$ to itself defined by $(\eta(v)(u), \xi(u)(v))=R(u, v)$. We suppose that these maps satisfy the invariance condition

$$
\xi(u)(v) \eta(v)(u)=u v \quad(\forall u, v \in L)
$$

Because the binary operation of $L$ is associative, (4.6) is equivalent to the invariance condition (4.5), and $(L, R)$ is an object of the category $\mathcal{D}$ as a result. From Theorem 4.7 (and its proof), this YB map $R$ is constructed by a ternary system (Definition 3.1) satisfying (3.5) and (3.6).

\section{$\S 5$. Proof of Theorem 4.7}

This section presents the proof of Theorem 4.7. We shall first define a functor $S: \mathcal{A} \rightarrow \mathcal{D}$. 
Lemma 5.1. Let $((L, \cdot),(M, \mu), \pi)$ and $\left(\left(L^{\prime}, .^{\prime}\right),\left(M^{\prime}, \mu^{\prime}\right), \pi^{\prime}\right)$ be elements of LMB. The following conditions are equivalent:

(1) $(L, M, \pi) \sim\left(L^{\prime}, M^{\prime}, \pi^{\prime}\right)$;

(2) $\left(R^{(L, M, \pi)}(\lambda) ; L, L,(\cdot)\right)=\left(R^{\left(L^{\prime}, M^{\prime}, \pi^{\prime}\right)}(\lambda) ; L^{\prime}, L^{\prime},\left(.^{\prime}\right)\right)(1.2)$; that is, $L=L^{\prime}$ as left quasigroups, and $R^{(L, M, \pi)}(\lambda)=R^{\left(L, M^{\prime}, \pi^{\prime}\right)}(\lambda)$ for all $\lambda \in L\left(=L^{\prime}\right)$.

Proof. On account of (3.2), the condition (2) is equivalent to the condition (3) below.

(3) $L=L^{\prime}$ as left quasigroups, and $\xi_{\lambda}^{(L, M, \pi)}(u)=\xi_{\lambda}^{\left(L, M^{\prime}, \pi^{\prime}\right)}(u)$ for all $\lambda, u \in L$.

We shall only show (1) from (3). It suffices to prove that the map $h=$ $\pi^{\prime} \pi^{-1}: M \rightarrow M^{\prime}$ is a homomorphism (4.1) of ternary systems.

Let $a, b$, and $c$ be elements of $M$. We define the elements $\lambda, u$, and $v$ of the left quasigroup $L$ by $\lambda=\pi^{-1}(a), u=\pi^{-1}(a) \backslash_{L} \pi^{-1}(b)$, and $v=$ $\pi^{-1}(b) \backslash_{L} \pi^{-1}(c)$. Here $\backslash_{L}$ is the left division (2.1) of $L$. Because of (3.1),

$$
\begin{aligned}
\pi^{-1}(\mu(a, b, c)) & =\lambda\left(\lambda \backslash_{L} \pi^{-1}(\mu(\pi(\lambda), \pi(\lambda u), \pi((\lambda u) v)))\right) \\
& =\lambda \xi_{\lambda}^{(L, M, \pi)}(u)(v) .
\end{aligned}
$$

It follows from the condition (3) that

$$
\pi^{-1}(\mu(a, b, c))=\pi^{\prime-1}\left(\mu^{\prime}(h(a), h(b), h(c))\right) .
$$

Hence, the map $h$ is a homomorphism of ternary systems.

Let $V=\left[\left(L_{V}, M, \pi\right)\right]$ be an object of the category $\mathcal{A}$. From Lemma 5.1, we can define the dynamical YB map $R^{V}(\lambda)$ associated with $L_{V}, L_{V}$, and (·), by using the dynamical YB map $R^{\left(L_{V}, M, \pi\right)}(\lambda)(3.3)$;

$$
R^{V}(\lambda)=R^{\left(L_{V}, M, \pi\right)}(\lambda) .
$$

Let $V$ be an object of the category $\mathcal{A}$. We define $S(V)$ by $S(V)=$ $\left(L_{V}, R^{V}(\lambda)\right)$.

Lemma 5.2. For $V \in O b(\mathcal{A}), S(V)$ is an object of the category $\mathcal{D}$.

Proof. The proof is immediate from (3.4) and Theorem 3.2.

Lemma 5.3. Let $V$ and $V^{\prime}$ be objects of the category $\mathcal{A}$. If $f: V \rightarrow$ $V^{\prime} \in \operatorname{Hom}(\mathcal{A})$, then $f$ is a morphism of the category $\mathcal{D}$ whose source and target are $S(V)$ and $S\left(V^{\prime}\right)$, respectively. 
Proof. Let $(L,(M, \mu), \pi)$ and $\left(L^{\prime},\left(M^{\prime}, \mu^{\prime}\right), \pi^{\prime}\right)$ be representatives of $V$ and $V^{\prime}$, respectively.

We shall demonstrate that $R^{\left(L^{\prime}, M^{\prime}, \pi^{\prime}\right)}(f(\lambda))(f \times f)=(f \times f) R^{(L, M, \pi)}(\lambda)$ for all $\lambda \in L$. Let $u$ and $v$ be elements of the left quasigroup $L$. Because the map $\pi^{\prime} f \pi^{-1}: M \rightarrow M^{\prime}$ is a homomorphism (4.1) of ternary systems (see Definition 4.1),

$$
\begin{aligned}
& f\left(\pi^{-1}(\mu(\pi(\lambda), \pi(\lambda u), \pi((\lambda u) v)))\right) \\
= & \pi^{\prime-1}\left(\mu^{\prime}\left(\pi^{\prime}(f(\lambda)), \pi^{\prime}(f(\lambda u)), \pi^{\prime}(f((\lambda u) v))\right)\right) .
\end{aligned}
$$

Since the map $f: L \rightarrow L^{\prime}$ is a homomorphism of left quasigroups, (3.1) and (5.2) induce that

$$
\begin{aligned}
f\left(\xi_{\lambda}^{(L, M, \pi)}(u)(v)\right) & =f(\lambda) \backslash_{L} f\left(\pi^{-1}(\mu(\pi(\lambda), \pi(\lambda u), \pi((\lambda u) v)))\right) \\
& =\xi_{f(\lambda)}^{\left(L^{\prime}, M^{\prime}, \pi^{\prime}\right)}(f(u))(f(v)) .
\end{aligned}
$$

The above equation and (3.2) lead to that

$$
f\left(\eta_{\lambda}^{(L, M, \pi)}(v)(u)\right)=\eta_{f(\lambda)}^{\left(L^{\prime}, M^{\prime}, \pi^{\prime}\right)}(f(u))(f(v)),
$$

because the map $f: L \rightarrow L^{\prime}$ is a homomorphism of left quasigroups.

From (5.3) and (5.4), $R^{\left(L^{\prime}, M^{\prime}, \pi^{\prime}\right)}(f(\lambda))(f \times f)=(f \times f) R^{(L, M, \pi)}(\lambda)$ for all $\lambda \in L$. Thus $f: S(V) \rightarrow S\left(V^{\prime}\right)$ is a morphism of the category $\mathcal{D}$.

For $f: V \rightarrow V^{\prime} \in \operatorname{Hom}(\mathcal{A})$, we define $S(f): S(V) \rightarrow S\left(V^{\prime}\right) \in \operatorname{Hom}(\mathcal{D})$ by $S(f)=f$.

Proposition 5.4. $\quad S$ is a functor from the category $\mathcal{A}$ to the category $\mathcal{D}$.

The next task is to introduce a functor $T: \mathcal{D} \rightarrow \mathcal{A}$. Let $V=(L, R(\lambda))$ be an object of the category $\mathcal{D}$. We define the maps $\xi_{\lambda}(u)$ and $\eta_{\lambda}(v)(\lambda, u, v \in L)$ from $L$ to $L$ by (4.4). Let $\mu_{L}$ denote the ternary operation on $L$ defined by

$$
\mu_{L}(a, b, c)=a \xi_{a}\left(a \backslash_{L} b\right)\left(b \backslash_{L} c\right) \quad(a, b, c \in L) .
$$

Lemma 5.5. The ternary operation $\mu_{L}$ (5.5) satisfies (3.5) and (3.6).

Proof. Let $\lambda$ be an element of the left quasigroup $L$. We define the maps $s(\lambda): L \times L \rightarrow L \times L$ and $s: L \times L \times L \rightarrow L \times L \times L$ as follows:

$$
\begin{aligned}
& s(\lambda)(a, b)=\left(\mu_{L}(\lambda, a, b), b\right) \quad(a, b \in L) ; \\
& s(a, b, c)=\left(a, \mu_{L}(a, b, c), c\right) \quad(a, b, c \in L) .
\end{aligned}
$$


On account of Lemma 3.3, it suffices to prove that the maps $s(\lambda)_{12}$ and $s$ satisfy the braid group relation (3.9).

Let $\sigma(\lambda)$ denote the map from $L \times L$ to itself defined by $\sigma(\lambda)=P_{L} R(\lambda)$. Here $P_{L}$ is the map (2.9). The maps $\sigma(\lambda)_{12}$ and $\sigma\left(\lambda L^{(1)}\right)_{23}$ are expressed as follows (cf. Lemma 3.5):

$$
\sigma(\lambda)_{12}=f_{\lambda}^{-1} s(\lambda)_{12} f_{\lambda} ; \quad \sigma\left(\lambda L^{(1)}\right)_{23}=f_{\lambda}^{-1} s f_{\lambda} .
$$

Here $f_{\lambda}$ is the bijection (3.10) (see Lemma 3.4).

Because $\sigma(\lambda)$ is a dynamical braiding map associated with $L, L$, and (·) (see Definition 2.8 and Proposition 2.9), the maps $s(\lambda)_{12}$ and $s$ satisfy (3.9). This completes the proof.

Corollary 5.6. The triplet $\left(L,\left(L, \mu_{L}\right), \mathrm{id}_{L}\right)$ is an element of the set $L M B$.

Let $V=(L, R(\lambda))$ be an object of the category $\mathcal{D}$. We define $T(V) \in$ $O b(\mathcal{A})$ by $T(V)=\left[\left(L,\left(L, \mu_{L}\right), \operatorname{id}_{L}\right)\right]$.

Lemma 5.7. Let $V$ and $V^{\prime}$ be objects of the category $\mathcal{D}$. If $f: V \rightarrow$ $V^{\prime} \in \operatorname{Hom}(\mathcal{D})$, then $f$ is a morphism of the category $\mathcal{A}$ whose source and target are $T(V)$ and $T\left(V^{\prime}\right)$, respectively.

Proof. Let $(L, R(\lambda))$ and $\left(L^{\prime}, R^{\prime}\left(\lambda^{\prime}\right)\right)$ denote the objects $V$ and $V^{\prime}$, respectively. We define the maps $\xi_{\lambda}(u): L \rightarrow L, \eta_{\lambda}(v): L \rightarrow L, \xi_{\lambda^{\prime}}^{\prime}\left(u^{\prime}\right): L^{\prime} \rightarrow L^{\prime}$, and $\eta_{\lambda^{\prime}}^{\prime}\left(v^{\prime}\right): L^{\prime} \rightarrow L^{\prime}\left(\lambda, u, v \in L, \lambda^{\prime}, u^{\prime}, v^{\prime} \in L^{\prime}\right)$ by $(4.4):\left(\eta_{\lambda}(v)(u), \xi_{\lambda}(u)(v)\right)=$ $R(\lambda)(u, v) ;\left(\eta_{\lambda^{\prime}}^{\prime}\left(v^{\prime}\right)\left(u^{\prime}\right), \xi_{\lambda^{\prime}}^{\prime}\left(u^{\prime}\right)\left(v^{\prime}\right)\right)=R^{\prime}\left(\lambda^{\prime}\right)\left(u^{\prime}, v^{\prime}\right)$. Let $\mu_{L}$ and $\mu_{L^{\prime}}$ denote the ternary operations on $L$ and $L^{\prime}$ defined by (5.5), respectively.

We shall show that $f:\left(L, \mu_{L}\right) \rightarrow\left(L^{\prime}, \mu_{L^{\prime}}\right)$ is a homomorphism (4.1) of ternary systems. By Definition 4.5, $R^{\prime}(f(\lambda))(f \times f)=(f \times f) R(\lambda)$ for all $\lambda \in L$. As a result, $f\left(\xi_{\lambda}(u)(v)\right)=\xi_{f(\lambda)}^{\prime}(f(u))(f(v))$ for all $\lambda, u, v \in L$. Because $f: L \rightarrow L^{\prime}$ is a homomorphism of left quasigroups, the above equation and the definition (5.5) of $\mu_{L}$ and $\mu_{L^{\prime}}$ induce that $f\left(\mu_{L}(a, b, c)\right)=\mu_{L^{\prime}}(f(a), f(b), f(c))$ for all $a, b, c \in L$. That is, $f:\left(L, \mu_{L}\right) \rightarrow\left(L^{\prime}, \mu_{L^{\prime}}\right)$ is a homomorphism of ternary systems.

Since $\left(L,\left(L, \mu_{L}\right), \operatorname{id}_{L}\right) \in T(V),\left(L^{\prime},\left(L^{\prime}, \mu_{L^{\prime}}\right), \operatorname{id}_{L^{\prime}}\right) \in T\left(V^{\prime}\right)$, and $_{\operatorname{id}_{L^{\prime}}}^{-1} f \mathrm{id}_{L}$ : $\left(L, \mu_{L}\right) \rightarrow\left(L^{\prime}, \mu_{L^{\prime}}\right)$ is a homomorphism of ternary systems, Remark 4.2 gives rise to that $f: T(V) \rightarrow T\left(V^{\prime}\right)$ is a morphism of the category $\mathcal{A}$.

For $f: V \rightarrow V^{\prime} \in \operatorname{Hom}(\mathcal{D})$, we define $T(f): T(V) \rightarrow T\left(V^{\prime}\right) \in \operatorname{Hom}(\mathcal{A})$ by $T(f)=f$. 
Proposition 5.8. $\quad T$ is a functor from the category $\mathcal{D}$ to the category $\mathcal{A}$.

Proof of Theorem 4.7. We shall only demonstrate that $T S(V)=V$ for $V \in O b(\mathcal{A})$.

Let $\left(L_{V},(M, \mu), \pi\right)$ be a representative of $V$. By the definitions, $S(V)=$ $\left(L_{V}, R^{\left(L_{V}, M, \pi\right)}(\lambda)\right)$ and $T S(V)=\left[\left(L_{V},\left(L_{V}, \mu_{L_{V}}\right), \operatorname{id}_{L_{V}}\right)\right]$ (see (5.1) and (5.5)).

For the proof, it suffices to show that $\left(L_{V},\left(L_{V}, \mu_{L_{V}}\right), \operatorname{id}_{L_{V}}\right) \sim\left(L_{V}, M, \pi\right)$. Let $a, b$, and $c$ be elements of $L_{V}$. From (3.1) and (5.5),

$$
\pi\left(\mu_{L_{V}}(a, b, c)\right)=\pi\left(a \xi_{a}^{\left(L_{V}, M, \pi\right)}\left(a \backslash_{L_{V}} b\right)\left(b \backslash_{L_{V}} c\right)\right)=\mu(\pi(a), \pi(b), \pi(c)) .
$$

Hence, the map $\pi \operatorname{id}_{L_{V}}^{-1}:\left(L_{V}, \mu_{L_{V}}\right) \rightarrow(M, \mu)$ is a homomorphism (4.1) of ternary systems, and consequently, $\left(L_{V},\left(L_{V}, \mu_{L_{V}}\right), \operatorname{id}_{L_{V}}\right) \sim\left(L_{V}, M, \pi\right)$.

\section{§6. Examples of Ternary Systems}

This section describes several ternary systems (Definition 3.1) satisfying (3.5) and (3.6). Later we shall characterize the dynamical YB maps $R^{(L, M, \pi)}(\lambda)$ (3.3) constructed by means of these ternary systems.

Example 6.1. Let $M$ be a nonempty set, and $f$ a map from the set $M$ to $M$. We define the ternary operations on $M$ by:

$$
\begin{aligned}
& \mu(a, b, c)=f(a) \quad(\forall a, b, c \in M) \\
& \mu(a, b, c)=f(c) \quad(\forall a, b, c \in M) .
\end{aligned}
$$

Each ternary system $(M, \mu)$ defined above satisfies (3.5) and (3.6).

Remark 6.2. Example 6.1 satisfying $f=\mathrm{id}_{M}$ produces degenerate YB maps in [1].

(1) Let $\mu$ denote the ternary operation on $L$ defined by $\mu(a, b, c)=c$. If $L$ is a left quasigroup together with the binary operation $u v:=v$, then $R^{\left(L,(L, \mu), \operatorname{id}_{L}\right)}(\lambda)(u, v)=(v, v)(\lambda, u, v \in L)$. This is the map $P_{L} \Delta_{2}$ in [1]. Here $P_{L}$ is the map (2.9).

(2) If $L=\left(L, \cdot, e_{L}\right)$ is a group and $\mu(a, b, c)=c(a, b, c \in L)$, then $R^{(L, M, \pi)}(\lambda)$ is the map $P_{L} \mu_{1}$ in [1]. If $L=\left(L, \cdot, e_{L}\right)$ is an abelian group and $\mu(a, b, c)=a$ $(a, b, c \in L)$, then $R^{(L, M, \pi)}(\lambda)$ is the map $P_{L} \mu_{2}$ in [1]. 
Example 6.3. $\quad$ Let $M$ be a nonempty set, and $f$ a map from $M$ to $M$ satisfying $f^{2}=f$. We define the ternary operation on $M$ by

$$
\mu(a, b, c)=f(b) \quad(\forall a, b, c \in M) .
$$

This ternary system $(M, \mu)$ satisfies (3.5) and (3.6).

Example 6.4. Let $M_{1}=\left(M_{1}, \mu_{1}\right)$ and $M_{2}=\left(M_{2}, \mu_{2}\right)$ be ternary systems satisfying (3.5) and (3.6). We denote by $M$ the direct product $M_{1} \times M_{2}$ of the sets $M_{1}$ and $M_{2}$; in addition, let us define the ternary operation $\mu$ on the set $M$ by

$$
\begin{aligned}
& \mu(a, b, c)=\left(\mu_{1}\left(a_{1}, b_{1}, c_{1}\right), \mu_{2}\left(a_{2}, b_{2}, c_{2}\right)\right) \\
& \left(a=\left(a_{1}, a_{2}\right), b=\left(b_{1}, b_{2}\right), c=\left(c_{1}, c_{2}\right) \in M=M_{1} \times M_{2}\right) .
\end{aligned}
$$

This ternary system $(M, \mu)$ satisfies (3.5) and (3.6).

We shall introduce three ternary operations $\mu_{1}^{G}(6.2), \mu_{2}^{G}(6.3)$, and $\mu_{3}^{G}$ (6.7) produced by left quasigroups.

Let $G=(G, *)$ be a left quasigroup (see Definition 2.1) satisfying that

$$
(a * c) \backslash_{G}((a * b) * c)=\left(a^{\prime} * c\right) \backslash_{G}\left(\left(a^{\prime} * b\right) * c\right) \quad\left(\forall a, a^{\prime}, b, c \in G\right) .
$$

Here $\backslash_{G}$ is the left division $(2.1)$ of $G$. Groups, the quasigroup $(\{1,2,3\}, *)$ in Example 2.3, and the left quasigroups having the right distributive law

$$
(x * y) * z=(x * z) *(y * z) \quad(\forall x, y, z \in G)
$$

satisfy (6.1) (see below the proof of Proposition 7.3). For distributive quasigroups, see [12, Section V.2].

We define the ternary operations $\mu_{1}^{G}$ and $\mu_{2}^{G}$ on the left quasigroup $G$ by:

$$
\begin{array}{ll}
\mu_{1}^{G}(a, b, c)=a *\left(b \backslash_{G} c\right) & (a, b, c \in G) ; \\
\mu_{2}^{G}(a, b, c)=c *\left(b \backslash_{G} a\right) & (a, b, c \in G) .
\end{array}
$$

Proposition 6.5. These ternary systems $\left(G, \mu_{1}^{G}\right)$ and $\left(G, \mu_{2}^{G}\right)$ satisfy (3.5) and (3.6).

Proof. We shall only prove that the ternary system $\left(G, \mu_{1}^{G}\right)$ satisfies (3.5) and (3.6).

The following lemma gives rise to (3.5). Its proof is immediate from (6.2).

Lemma 6.6. For $a, b, c, d \in G, \mu_{1}^{G}\left(a, b, \mu_{1}^{G}(b, c, d)\right)=\mu_{1}^{G}(a, c, d)$. 
We shall prove (3.6). In view of (6.2),

LHS of (3.6)

$$
\begin{aligned}
& =\left(a *\left(b \backslash_{G} c\right)\right) *\left(c \backslash_{G} d\right) \\
& =\left(a *\left(c \backslash_{G} d\right)\right) *\left(\left(a *\left(c \backslash_{G} d\right)\right) \backslash_{G}\left(\left(a *\left(b \backslash_{G} c\right)\right) *\left(c \backslash_{G} d\right)\right)\right),
\end{aligned}
$$

RHS of (3.6)

$$
\begin{aligned}
& =\left(a *\left(c \backslash_{G} d\right)\right) *\left(\left(b *\left(c \backslash_{G} d\right)\right) \backslash_{G} d\right) \\
& =\left(a *\left(c \backslash_{G} d\right)\right) *\left(\left(b *\left(c \backslash_{G} d\right)\right) \backslash_{G}\left(\left(b *\left(b \backslash_{G} c\right)\right) *\left(c \backslash_{G} d\right)\right)\right) .
\end{aligned}
$$

The right-hand-sides of the above equations are the same, because of (6.1).

Remark 6.7. Every dynamical YB map $R^{(G)}(\lambda)(2.6)$ constructed in the work [13] is produced by the ternary system $\left(G, \mu_{1}^{G}\right)(6.2)$. Let $L$ be a loop, $G=\left(G, *, e_{G}\right)$ a group, and $\pi: L \rightarrow G$ a bijection satisfying $\pi\left(e_{L}\right)=e_{G}$. Here $e_{G}$ is the unit element of the group $G$. By the definitions (2.2) of the maps $\theta(u)$ and $\theta(u)^{-1}$, the map $\xi_{\lambda}^{(G)}(u)(2.3)(\lambda, u \in L)$ is expressed as

$$
\xi_{\lambda}^{(G)}(u)(v)=\lambda \backslash_{L} \pi^{-1}\left(\mu_{1}^{G}(\pi(\lambda), \pi(\lambda u), \pi((\lambda u) v))\right) \quad(v \in L) .
$$

On account of (2.3), (2.4), (3.1), and (3.2), (6.4) induces that all the dynamical YB maps $R^{(G)}(\lambda)$ are constructed by means of the ternary systems $\left(G, \mu_{1}^{G}\right)$.

Next task is to define the ternary operation $\mu_{3}^{G}(6.7)$.

Let $G=(G, *)$ be a left quasigroup. We suppose that $G$ satisfies the following for all $a, b, c, d \in G$ :

$$
\begin{aligned}
& (b * c) *\left(a \backslash_{G}\left((a * c) *\left((b * c) \backslash_{G}(b * d)\right)\right)\right) \\
= & b *\left(a \backslash_{G}((a * c) * d)\right) ; \\
& (a * c) *\left((b * c) \backslash_{G}(b * d)\right) \\
= & ((a * c) * d) *\left(\left(b *\left(a \backslash_{G}((a * c) * d)\right)\right) \backslash_{G}(b * d)\right) .
\end{aligned}
$$

If $G$ is a group, $G$ satisfies (6.5) and (6.6).

Let $\mu_{3}^{G}$ denote the ternary operation on the set $G$ defined by

$$
\mu_{3}^{G}(a, b, c)=b *\left(a \backslash_{G} c\right) \quad(a, b, c \in G) .
$$

Proposition 6.8. This ternary system $\left(G, \mu_{3}^{G}\right)$ satisfies (3.5) and (3.6).

Proof. We shall only prove that the ternary system $\left(G, \mu_{3}^{G}\right)$ satisfies (3.5). 
In view of (6.7),

LHS of (3.5)

$$
\begin{aligned}
= & \left(b *\left(a \backslash_{G} c\right)\right) *\left(a \backslash_{G}\left(c *\left(\left(b *\left(a \backslash_{G} c\right)\right) \backslash_{G} d\right)\right)\right) ; \\
& \operatorname{RHS} \text { of }(3.5) \\
= & b *\left(a \backslash_{G}\left(c *\left(b \backslash_{G} d\right)\right)\right) \\
= & b *\left(a \backslash_{G}\left(\left(a *\left(a \backslash_{G} c\right)\right) *\left(b \backslash_{G} d\right)\right)\right) .
\end{aligned}
$$

The right-hand-sides of the above equations are the same, because of (6.5).

Final task in this section is to characterize the dynamical YB map $R^{(L, M, \pi)}(\lambda)$ (3.3) that the ternary systems (6.2), (6.3), and (6.7) define.

Let $\mathcal{A}_{1}$ denote the subcategory of the category $\mathcal{A}$ whose objects and morphisms are defined as follows: $V \in O b(\mathcal{A})$ is an object of $\mathcal{A}_{1}$, iff there exists a representative $(L,(M, \mu), \pi)$ of $V$ such that the ternary operation $\mu$ on $M$ satisfies

$$
\begin{aligned}
& \mu(a, b, \mu(b, c, d))=\mu(a, c, d) \quad(\forall a, b, c, d \in M), \\
& \mu(a, a, b)=b \quad(\forall a, b \in M) ;
\end{aligned}
$$

$f: V \rightarrow V^{\prime} \in \operatorname{Hom}(\mathcal{A})$ is a morphism of $\mathcal{A}_{1}$, iff $V, V^{\prime} \in O b\left(\mathcal{A}_{1}\right)$.

Let $L$ be a left quasigroup, $G$ a left quasigroup satisfying (6.1), and $\pi$ a (set-theoretical) bijection from $L$ to $G$. $\left[\left(L,\left(G, \mu_{1}^{G}\right), \pi\right)\right]$ is an object of the category $\mathcal{A}$ because of Proposition 6.5. Moreover,

Proposition 6.9. $\left[\left(L,\left(G, \mu_{1}^{G}\right), \pi\right)\right]$ is an object of the category $\mathcal{A}_{1}$.

Proof. The proof is immediate from (6.2) (see Lemma 6.6).

Conversely, every object of the category $\mathcal{A}_{1}$ is expressed by means of the ternary system $\left(G, \mu_{1}^{G}\right)$.

Proposition 6.10. If $V \in O b\left(\mathcal{A}_{1}\right)$, then there exist a left quasigroup $(G, *)$ satisfying $(6.1)$ and a bijection $\pi^{\prime}: L_{V} \rightarrow G$ such that $V=\left[\left(L_{V},(G\right.\right.$, $\left.\left.\left.\mu_{1}^{G}\right), \pi^{\prime}\right)\right]$.

Proof. We denote by $L$ the left quasigroup $L_{V}$. Since $V \in O b\left(\mathcal{A}_{1}\right)$, there exists a representative $(L,(M, \mu), \pi)$ of $V$ such that the ternary operation $\mu$ on $M$ satisfies (6.8) and (6.9). 
We fix any element $\lambda \in L$. Let $\alpha$ and $\beta$ denote the following binary operations on $L$ : for $u, v \in L$,

$$
\begin{aligned}
& \alpha(u, v)=\lambda \backslash_{L} \pi^{-1}(\mu(\pi(\lambda), \pi(\lambda u), \pi(\lambda v))) ; \\
& \beta(u, v)=\lambda \backslash_{L} \pi^{-1}(\mu(\pi(\lambda u), \pi(\lambda), \pi(\lambda v))) .
\end{aligned}
$$

Lemma 6.11. For all $u, v \in L, \beta(u, \alpha(u, v))=v$ and $\alpha(u, \beta(u, v))=v$.

Proof. The proof is immediate from (6.8) and (6.9).

We denote by $G$ and $(*)$ the set $L$ and the binary operation $\beta$ on the set $G(=L)$, respectively. The above lemma gives rise to that $G=(G, *)$ is a left quasigroup. The left division on $G$ is the binary operation $\alpha ; a \backslash_{G} c=\alpha(a, c)$ $(a, c \in G)$.

Lemma 6.12. The left quasigroup G satisfies (6.1).

Proof. Because of (6.8), (6.10), and (6.11),

$$
a *\left(b \backslash_{G} c\right)=\lambda \backslash_{L} \pi^{-1}(\mu(\pi(\lambda a), \pi(\lambda b), \pi(\lambda c))) \quad(\forall a, b, c \in G) .
$$

Let $a, a^{\prime}, b$, and $c$ be elements of $G(=L)$. In view of (6.12),

$$
\begin{aligned}
& (a * b) * c \\
= & \left(a *\left(a^{\prime} \backslash_{G}\left(a^{\prime} * b\right)\right)\right) *\left(\left(a^{\prime} * b\right) \backslash_{G}\left(\left(a^{\prime} * b\right) * c\right)\right) \\
= & \lambda \backslash_{L} \pi^{-1}\left(\mu\left(\mu\left(\pi(\lambda a), \pi\left(\lambda a^{\prime}\right), \pi\left(\lambda\left(a^{\prime} * b\right)\right)\right), \pi\left(\lambda\left(a^{\prime} * b\right)\right), \pi\left(\lambda\left(\left(a^{\prime} * b\right) * c\right)\right)\right)\right) ; \\
& (a * c) *\left(\left(a^{\prime} * c\right) \backslash_{G}\left(\left(a^{\prime} * b\right) * c\right)\right) \\
= & \left(a *\left(a^{\prime} \backslash_{G}\left(a^{\prime} *\left(\left(a^{\prime} * b\right) \backslash_{G}\left(\left(a^{\prime} * b\right) * c\right)\right)\right)\right)\right) * \\
& *\left(\left(a^{\prime} *\left(\left(a^{\prime} * b\right) \backslash_{G}\left(\left(a^{\prime} * b\right) * c\right)\right)\right) \backslash_{G}\left(\left(a^{\prime} * b\right) * c\right)\right) \\
= & \lambda \backslash_{L} \pi^{-1}\left(\mu \left(\mu\left(\pi(\lambda a), \pi\left(\lambda a^{\prime}\right), \mu\left(\pi\left(\lambda a^{\prime}\right), \pi\left(\lambda\left(a^{\prime} * b\right)\right), \pi\left(\lambda\left(\left(a^{\prime} * b\right) * c\right)\right)\right)\right),\right.\right. \\
& \left.\left.\mu\left(\pi\left(\lambda a^{\prime}\right), \pi\left(\lambda\left(a^{\prime} * b\right)\right), \pi\left(\lambda\left(\left(a^{\prime} * b\right) * c\right)\right)\right), \pi\left(\lambda\left(\left(a^{\prime} * b\right) * c\right)\right)\right)\right) .
\end{aligned}
$$

With the aid of (3.6),

$$
(a * b) * c=(a * c) *\left(\left(a^{\prime} * c\right) \backslash_{G}\left(\left(a^{\prime} * b\right) * c\right)\right)
$$

for all $a, a^{\prime}, b, c \in G$. This is equivalent to (6.1).

Let $\pi^{\prime}$ denote the map from $L$ to $G(=L)$ defined by $\pi^{\prime}(u)=\lambda \backslash_{L} u(u \in L)$. 
Lemma 6.13. The map $\pi^{\prime}$ is bijective; $\pi^{\prime-1}(a)=\lambda a(a \in G)$.

Finally, we shall demonstrate that $V=\left[\left(L,\left(G, \mu_{1}^{G}\right), \pi^{\prime}\right)\right]$. From (6.12),

$$
\pi^{\prime} \pi^{-1}\left(\mu\left(\pi \pi^{\prime-1}(a), \pi \pi^{\prime-1}(b), \pi \pi^{\prime-1}(c)\right)\right)=a *\left(b \backslash_{G} c\right)=\mu_{1}^{G}(a, b, c) .
$$

Hence, the map $\pi \pi^{\prime-1}:\left(G, \mu_{1}^{G}\right) \rightarrow M$ is a homomorphism (4.1) of ternary systems. As a result, $(L, M, \pi) \sim\left(L,\left(G, \mu_{1}^{G}\right), \pi^{\prime}\right)$; that is, $V=\left[\left(L,\left(G, \mu_{1}^{G}\right), \pi^{\prime}\right)\right]$.

This completes the proof of Proposition 6.10.

We denote by $\mathcal{D}_{1}$ the subcategory of the category $\mathcal{D}$ whose objects and morphisms are defined as follows: $V=(L, R(\lambda)) \in O b(\mathcal{D})$ is an object of $\mathcal{D}_{1}$, iff the map $\xi_{\lambda}(u)(\lambda, u \in L)$ (4.4) satisfies

$$
\begin{aligned}
& \xi_{\lambda}(u) \xi_{\lambda u}(v)=\xi_{\lambda}\left(\lambda \backslash_{L}((\lambda u) v)\right) \quad(\forall \lambda, u, v \in L), \\
& \xi_{\lambda}\left(\lambda \backslash_{L} \lambda\right)=\operatorname{id}_{L} \quad(\forall \lambda \in L) ;
\end{aligned}
$$

$f: V \rightarrow V^{\prime} \in \operatorname{Hom}(\mathcal{D})$ is a morphism of $\mathcal{D}_{1}$, iff $V, V^{\prime} \in O b\left(\mathcal{D}_{1}\right)$.

The functors $S: \mathcal{A} \rightarrow \mathcal{D}$ and $T: \mathcal{D} \rightarrow \mathcal{A}$ induce the following.

Proposition 6.14. The category $\mathcal{A}_{1}$ is isomorphic to the category $\mathcal{D}_{1}$.

Proof. The proof is straightforward.

Let us introduce subcategories $\mathcal{A}_{2}$ and $\mathcal{A}_{3}$ (resp. $\mathcal{D}_{2}$ and $\mathcal{D}_{3}$ ) of the category $\mathcal{A}$ (resp. $\mathcal{D})$, which characterize the dynamical YB maps $R^{(L, M, \pi)}(\lambda)(3.3)$ constructed by means of the ternary systems $(6.3)$ and $(6.7): V \in O b(\mathcal{A})$ is an object of $\mathcal{A}_{2}$, iff there exists a representative $(L,(M, \mu), \pi)$ of $V$ such that the ternary operation $\mu$ on $M$ satisfies

$$
\begin{aligned}
& \mu(\mu(a, b, c), c, d)=\mu(a, b, d) \quad(\forall a, b, c, d \in M), \\
& \mu(a, b, b)=a \quad(\forall a, b \in M) ;
\end{aligned}
$$

$f: V \rightarrow V^{\prime} \in \operatorname{Hom}(\mathcal{A})$ is a morphism of $\mathcal{A}_{2}$, iff $V, V^{\prime} \in O b\left(\mathcal{A}_{2}\right) ; V \in O b(\mathcal{A})$ is an object of $\mathcal{A}_{3}$, iff there exists a representative $(L,(M, \mu), \pi)$ of $V$ such that the ternary operation $\mu$ on $M$ satisfies

$$
\begin{aligned}
& \mu(a, b, c)=\mu(d, b, \mu(a, d, c)) \quad(\forall a, b, c, d \in M), \\
& \mu(a, a, b)=b \quad(\forall a, b \in M) ;
\end{aligned}
$$

$f: V \rightarrow V^{\prime} \in \operatorname{Hom}(\mathcal{A})$ is a morphism of $\mathcal{A}_{3}$, iff $V, V^{\prime} \in O b\left(\mathcal{A}_{3}\right) ; V=$ $(L, R(\lambda)) \in O b(\mathcal{D})$ is an object of $\mathcal{D}_{2}$, iff the maps $\xi_{\lambda}(u)$ and $\eta_{\lambda}(v)(\lambda, u, v \in L)$ 
(4.4) satisfy

$$
\begin{aligned}
& \left(\lambda \xi_{\lambda}(u)(v)\right) \xi_{\lambda \xi_{\lambda}(u)(v)}\left(\eta_{\lambda}(v)(u)\right)(w) \\
= & \lambda \xi_{\lambda}(u)\left((\lambda u) \backslash_{L}(((\lambda u) v) w)\right) \quad(\forall \lambda, u, v, w \in L), \\
& \xi_{\lambda}(u)\left((\lambda u) \backslash_{L}(\lambda u)\right)=\lambda \backslash_{L} \lambda \quad(\forall \lambda, u \in L) ;
\end{aligned}
$$

$f: V \rightarrow V^{\prime} \in \operatorname{Hom}(\mathcal{D})$ is a morphism of $\mathcal{D}_{2}$, iff $V, V^{\prime} \in O b\left(\mathcal{D}_{2}\right) ; V=$ $(L, R(\lambda)) \in O b(\mathcal{D})$ is an object of $\mathcal{D}_{3}$, iff the map $\xi_{\lambda}(u)(\lambda, u \in L)(4.4)$ satisfies

$$
\begin{aligned}
& \lambda \xi_{\lambda}(v)\left((\lambda v) \backslash_{L}\left((\lambda u) \xi_{\lambda u}\left((\lambda u) \backslash_{L} \lambda\right)(w)\right)\right) \\
= & (\lambda u) \xi_{\lambda u}\left((\lambda u) \backslash_{L}(\lambda v)\right)\left((\lambda v) \backslash_{L}(\lambda w)\right) \quad(\forall \lambda, u, v, w \in L), \\
& \xi_{\lambda}\left(\lambda \backslash_{L} \lambda\right)=\operatorname{id}_{L} \quad(\forall \lambda \in L) ;
\end{aligned}
$$

$f: V \rightarrow V^{\prime} \in \operatorname{Hom}(\mathcal{D})$ is a morphism of $\mathcal{D}_{3}$, iff $V, V^{\prime} \in O b\left(\mathcal{D}_{3}\right)$.

The functors $S: \mathcal{A} \rightarrow \mathcal{D}$ and $T: \mathcal{D} \rightarrow \mathcal{A}$ give rise to the following proposition.

Proposition 6.15. The categories $\mathcal{A}_{2}$ and $\mathcal{A}_{3}$ are isomorphic to the categories $\mathcal{D}_{2}$ and $\mathcal{D}_{3}$, respectively.

The proof of the following proposition is immediate from (6.3).

Proposition 6.16. Let $L$ be a left quasigroup, $G$ a left quasigroup satisfying (6.1), and $\pi$ a (set-theoretical) bijection from $L$ to $G$. Then $\left[\left(L,\left(G, \mu_{2}^{G}\right)\right.\right.$, $\pi)$ ] is an object of the category $\mathcal{A}_{2}$.

Proposition 6.17. If $V \in O b\left(\mathcal{A}_{2}\right)$, then there exist a left quasigroup $(G, *)$ satisfying $(6.1)$ and a bijection $\pi^{\prime}: L_{V} \rightarrow G$ such that $V=\left[\left(L_{V},\left(G, \mu_{2}^{G}\right)\right.\right.$, $\left.\left.\pi^{\prime}\right)\right]$.

Proof. The proof is similar to that of Proposition 6.10. For the reason that $V \in O b\left(\mathcal{A}_{2}\right)$, there exists a representative $\left(L_{V},(M, \mu), \pi\right)$ of $V$ such that the ternary operation $\mu$ on $M$ satisfies (6.13) and (6.14).

Let $G$ denote the set $L_{V}$. We fix any element $\lambda \in G\left(=L_{V}\right)$. Let us define the binary operation $*$ on $G\left(=L_{V}\right)$ by

$$
a * b=\lambda \backslash_{L_{V}} \pi^{-1}(\mu(\pi(\lambda b), \pi(\lambda), \pi(\lambda a))) \quad(a, b \in G) .
$$

Then $(G, *)$ is a left quasigroup; the left division $\backslash_{G}$ is as follows.

$$
a \backslash_{G} c=\lambda \backslash_{L_{V}} \pi^{-1}(\mu(\pi(\lambda c), \pi(\lambda a), \pi(\lambda))) \quad\left(a, c \in G\left(=L_{V}\right)\right) .
$$


The following equation induces that $(G, *)$ satisfies (6.1) (cf. Lemma 6.12).

$$
c *\left(b \backslash_{G} a\right)=\lambda \backslash_{L_{V}} \pi^{-1}(\mu(\pi(\lambda a), \pi(\lambda b), \pi(\lambda c))) \quad(\forall a, b, c \in G) .
$$

Moreover, we denote by $\pi^{\prime}$ the bijection from $L_{V}$ to $G\left(=L_{V}\right)$ defined by $\pi^{\prime}(u)=\lambda \backslash_{L_{V}} u\left(u \in L_{V}\right)$. The triplet $\left(L_{V},\left(G, \mu_{2}^{G}\right), \pi^{\prime}\right)$ is a representative of $V$; that is, $V=\left[\left(L_{V},\left(G, \mu_{2}^{G}\right), \pi^{\prime}\right)\right]$.

Let $L$ be a left quasigroup, $G$ a left quasigroup satisfying (6.5) and (6.6), and $\pi$ a (set-theoretical) bijection from $L$ to $G$. Then $\left[\left(L,\left(G, \mu_{3}^{G}\right), \pi\right)\right]$ is an object of the category $\mathcal{A}$ by virtue of Proposition 6.8 .

Proposition 6.18. $\left[\left(L,\left(G, \mu_{3}^{G}\right), \pi\right)\right]$ is an object of the category $\mathcal{A}_{3}$.

Proposition 6.19. If $V \in O b\left(\mathcal{A}_{3}\right)$, then there exist a left quasigroup $(G, *)$ satisfying $(6.5)$ and $(6.6)$, and a bijection $\pi^{\prime}: L_{V} \rightarrow G$ such that $V=$ $\left[\left(L_{V},\left(G, \mu_{3}^{G}\right), \pi^{\prime}\right)\right]$.

Proof. Because $V \in O b\left(\mathcal{A}_{3}\right)$, there exists a representative $\left(L_{V},(M, \mu), \pi\right)$ of $V$ such that the ternary operation $\mu$ on $M$ satisfies (6.15) and (6.16).

Let $G$ denote the set $L_{V}$. We fix any element $\lambda \in G\left(=L_{V}\right)$. Let us define the binary operation $*$ on $G\left(=L_{V}\right)$ by

$$
a * b=\lambda \backslash_{L_{V}} \pi^{-1}(\mu(\pi(\lambda), \pi(\lambda a), \pi(\lambda b))) \quad(a, b \in G) .
$$

Then $(G, *)$ is a left quasigroup satisfying (6.5) and (6.6); the left division $\backslash_{G}$ is as follows.

$$
a \backslash_{G} c=\lambda \backslash_{L_{V}} \pi^{-1}(\mu(\pi(\lambda a), \pi(\lambda), \pi(\lambda c))) \quad\left(a, c \in G\left(=L_{V}\right)\right) .
$$

The proof of (6.5) and (6.6) is due to the following (cf. Lemma 6.12).

$$
b *\left(a \backslash_{G} c\right)=\lambda \backslash_{L_{V}} \pi^{-1}(\mu(\pi(\lambda a), \pi(\lambda b), \pi(\lambda c))) \quad(\forall a, b, c \in G) .
$$

In addition, we denote by $\pi^{\prime}$ the bijection from $L_{V}$ to $G\left(=L_{V}\right)$ defined by $\pi^{\prime}(u)=\lambda \backslash_{L_{V}} u\left(u \in L_{V}\right)$.

These $(G, *)$ and $\pi^{\prime}$ are what we desire.

\section{§7. Unitary Condition}

Let $(L, M, \pi)$ be an element of $L M B$. In this section, we discuss the unitary condition (2.8) of the dynamical YB map $R^{(L, M, \pi)}(\lambda)(3.3)$. 
Proposition 7.1. The dynamical $Y B$ map $R^{(L, M, \pi)}(\lambda)$ satisfies the unitary condition, if and only if the ternary operation $\mu$ on $M$ satisfies that

$$
\mu(a, \mu(a, b, c), c)=b \quad(\forall a, b, c \in M) .
$$

Proof. Let $\lambda$ be an element of the left quasigroup $L$. We define the map $\tilde{f}_{\lambda}: L \times L \rightarrow L \times L$ by

$$
\tilde{f}_{\lambda}(u, v)=(\lambda u,(\lambda u) v) \quad(u, v \in L) .
$$

Lemma 7.2. The map $\tilde{f}_{\lambda}$ is bijective; $\tilde{f}_{\lambda}^{-1}(u, v)=\left(\lambda \backslash_{L} u, u \backslash_{L} v\right)(u, v$ $\in L)$. Here $\backslash_{L}$ is the left division (2.1) of $L$.

By using the maps $P_{L}(2.9), s(\pi(\lambda))(3.7), \tilde{f}_{\lambda}(7.2)$, and $\tilde{f}_{\lambda}^{-1}$, the dynamical YB map $R^{(L, M, \pi)}(\lambda)$ is expressed as follows.

$$
R^{(L, M, \pi)}(\lambda)=P_{L} \tilde{f}_{\lambda}^{-1}\left(\pi^{-1} \times \pi^{-1}\right) s(\pi(\lambda))(\pi \times \pi) \tilde{f}_{\lambda} .
$$

Since the map $\pi: L \rightarrow M$ is bijective, the unitary condition of the dynamical YB map $R^{(L, M, \pi)}(\lambda)$ is equivalent to that $s(a)^{2}=\operatorname{id}_{M \times M}$ for all $a \in M$.

The rest of the proof is immediate from the definition (3.7) of the map $s(a)$.

Let $M$ denote one of the ternary systems in Section 6. By using Proposition 7.1 , we shall clarify a necessary and sufficient condition for the dynamical YB map $R^{(L, M, \pi)}(\lambda)(3.3)$ to satisfy the unitary condition.

Let us suppose that $M$ is a ternary system in Example 6.1. From Proposition 7.1, the dynamical YB map $R^{(L, M, \pi)}(\lambda)$ does not satisfy the unitary condition, unless $|M|=1$. If $|M|=1$, then the dynamical YB map $R^{(L, M, \pi)}(\lambda)=$ $\operatorname{id}_{L \times L}$; hence, it is trivial that the dynamical YB map $R^{(L, M, \pi)}(\lambda)$ satisfies the unitary condition.

Next we suppose that $M$ is a ternary system in Example 6.3. The dynamical YB map $R^{(L, M, \pi)}(\lambda)$ satisfies the unitary condition, if and only if the map $f$ is the identity map id $\operatorname{id}_{M}$. If $f=\operatorname{id}_{M}$, then $R^{(L, M, \pi)}(\lambda)=P_{L}(2.9)$.

Proposition 7.3. Let $M$ be a ternary system $\left(G, \mu_{1}^{G}\right)(6.2)$ or $\left(G, \mu_{2}^{G}\right)$ (6.3). The dynamical $Y B$ map $R^{(L, M, \pi)}(\lambda)$ satisfies the unitary condition, if and only if

$$
(a * b) * c=(a * c) * b \quad(\forall a, b, c \in G) .
$$


Proof. Let $G=(G, *)$ be a left quasigroup satisfying (6.1). We shall only show this proposition in the case that $M=\left(G, \mu_{1}^{G}\right)$.

Let us suppose that the dynamical YB map $R^{(L, M, \pi)}(\lambda)$ satisfies the unitary condition. It follows from (6.2) and Proposition 7.1 that

$$
\begin{aligned}
a *\left((a * c) \backslash_{G}((a * b) * c)\right) & =\mu_{1}^{G}\left(a, \mu_{1}^{G}(a, a * b,(a * b) * c),(a * b) * c\right) \\
& =a * b \quad(\forall a, b, c \in G) .
\end{aligned}
$$

This is equivalent to (7.4), since $G$ is a left quasigroup.

Conversely, we suppose that Eq. (7.4) holds. With the aid of (6.2),

$$
\begin{aligned}
\operatorname{LHS} \text { of }(7.1) & =a *\left(\left(a *\left(b \backslash_{G} c\right)\right) \backslash_{G} c\right) \\
& =a *\left(\left(a *\left(b \backslash_{G} c\right)\right) \backslash_{G}\left(\left(a *\left(a \backslash_{G} b\right)\right) *\left(b \backslash_{G} c\right)\right)\right)
\end{aligned}
$$

for all $a, b, c \in G$. By virtue of (7.4),

$$
\begin{aligned}
\operatorname{RHS} \text { of }(7.5) & =a *\left(\left(a *\left(b \backslash_{G} c\right)\right) \backslash_{G}\left(\left(a *\left(b \backslash_{G} c\right)\right) *\left(a \backslash_{G} b\right)\right)\right) \\
& =b
\end{aligned}
$$

Because of Proposition 7.1, the dynamical YB map $R^{(L, M, \pi)}(\lambda)$ satisfies the unitary condition.

Let $G$ be an abelian group or the quasigroup $(\{1,2,3\}, *)$ in Example 2.3. This $G$ satisfies (7.4) (the proof is straightforward); in addition, (7.4) induces that $G$ satisfies (6.1).

Remark 7.4. If the left quasigroup $G$ is a group, then (7.4) is equivalent to that the group $G$ is abelian. Hence, Remark 6.7 and Proposition 7.3 reproduce Theorem 2.7 .

The proof of the proposition below is similar to that of Proposition 7.3.

Proposition 7.5. Let $M$ be a ternary system $\left(G, \mu_{3}^{G}\right)(6.7)$. The $d y$ namical YB map $R^{(L, M, \pi)}(\lambda)$ satisfies the unitary condition, if and only if

$$
(a * b) * b=a \quad(\forall a, b \in G) .
$$

\section{$\S 8 . \quad$ IRF-IRF Correspondence}

Let $L_{i}=\left(L_{i}, \bullet_{i}\right)(i=1,2)$ be left quasigroups (see Definition 2.1), $M=$ $(M, \mu)$ a ternary system (Definition 3.1 ) satisfying (3.5) and (3.6), and $\pi_{i}$ : 
$L_{i} \rightarrow M(i=1,2)$ bijections. Let $\lambda_{1}$ and $\lambda_{2}$ be elements of the left quasigroups $L_{1}$ and $L_{2}$, respectively.

To end this paper, we establish a correspondence between two dynamical YB maps $R^{\left(L_{1}, M, \pi_{1}\right)}\left(\lambda_{1}\right)$ and $R^{\left(L_{2}, M, \pi_{2}\right)}\left(\lambda_{2}\right)$ (3.3) called an IRF-IRF correspondence (Proposition 8.1). Let $\tilde{f}_{\lambda_{i}}^{(i)}(i=1,2)$ denote the map from $L_{i} \times L_{i}$ to itself defined by (7.2). By means of these maps, we define the map $J\left(\lambda_{1}\right)$ : $L_{1} \times L_{1} \rightarrow L_{2} \times L_{2}$ as follows.

$$
J\left(\lambda_{1}\right)=\tilde{f}_{\pi_{2}^{-1} \pi_{1}\left(\lambda_{1}\right)}^{(2)-1}\left(\pi_{2}^{-1} \pi_{1} \times \pi_{2}^{-1} \pi_{1}\right) \tilde{f}_{\lambda_{1}}^{(1)} P_{L_{1}} .
$$

Here $P_{L_{1}}$ is the map (2.9). Let us define the maps $R^{\left(L_{2}, M, \pi_{2}\right) 21}\left(\lambda_{2}\right): L_{2} \times$ $L_{2} \rightarrow L_{2} \times L_{2}$ and $J^{21}\left(\lambda_{1}\right): L_{1} \times L_{1} \rightarrow L_{2} \times L_{2}$ by $R^{\left(L_{2}, M, \pi_{2}\right) 21}\left(\lambda_{2}\right)=$ $P_{L_{2}} R^{\left(L_{2}, M, \pi_{2}\right)}\left(\lambda_{2}\right) P_{L_{2}}$ and $J^{21}\left(\lambda_{1}\right)=P_{L_{2}} J\left(\lambda_{1}\right) P_{L_{1}}$.

Eq. (7.3) plays an essential role in the proof of Proposition 8.1. $J^{21}\left(\lambda_{1}\right)$.

Proposition 8.1. $\quad R^{\left(L_{1}, M, \pi_{1}\right)}\left(\lambda_{1}\right)=J\left(\lambda_{1}\right)^{-1} R^{\left(L_{2}, M, \pi_{2}\right) 21}\left(\pi_{2}^{-1} \pi_{1}\left(\lambda_{1}\right)\right)$

Proof. Let $a$ denote the element $\pi_{1}\left(\lambda_{1}\right)$ of the ternary system $M$. From $(7.3)$,

$$
s(a)=\left(\pi_{i} \times \pi_{i}\right) \tilde{f}_{\pi_{i}^{-1}(a)}^{(i)} P_{L_{i}} R^{\left(L_{i}, M, \pi_{i}\right)}\left(\pi_{i}^{-1}(a)\right) \tilde{f}_{\pi_{i}^{-1}(a)}^{(i)-1}\left(\pi_{i}^{-1} \times \pi_{i}^{-1}\right)
$$

for $i=1,2$. This equation immediately induces Proposition 8.1.

This IRF-IRF correspondence is said to be a vertex-IRF correspondence, iff the dynamical YB map $R^{\left(L_{2}, M, \pi_{2}\right)}\left(\lambda_{2}\right)$ is independent of the dynamical parameter $\lambda_{2}$; hence, $R^{\left(L_{2}, M, \pi_{2}\right)}\left(\lambda_{2}\right)$ is a YB map. We denote by $R^{\left(L_{2}, M, \pi_{2}\right)}$ the YB map $R^{\left(L_{2}, M, \pi_{2}\right)}\left(\lambda_{2}\right)$. The vertex-IRF correspondence is as follows (cf. [4, Definition 5.4] and $[9,(4.10)])$.

$$
R^{\left(L_{1}, M, \pi_{1}\right)}\left(\lambda_{1}\right)=J\left(\lambda_{1}\right)^{-1} R^{\left(L_{2}, M, \pi_{2}\right) 21} J^{21}\left(\lambda_{1}\right) .
$$

Remark 8.2. The maps $J\left(\lambda_{1}\right)$ and $R^{\left(L_{1}, M, \pi_{1}\right)}\left(\lambda_{1}\right)$ correspond to the fusion matrix and the exchange matrix, respectively (see [4, Sections 5.1 and 5.2] and $[9$, Sections 3 and 4$]$ ).

Let $V=(L, R(\lambda))$ be an object of the category $\mathcal{D}_{1}$. We shall discuss a vertex-IRF correspondence whose IRF part is this dynamical YB map $R(\lambda)$.

Proposition 8.3. The dynamical $Y B$ map $R(\lambda)$ has at least one vertexIRF correspondence. 
Proof. From Propositions 6.10 and 6.14, there exist a left quasigroup $(G, *)$ satisfying $(6.1)$ and a bijection $\pi: L \rightarrow G$ such that $R(\lambda)=R^{\left(L,\left(G, \mu_{1}^{G}\right), \pi\right)}(\lambda)$ (3.3).

Let $\circ$ denote the binary operation on the set $L$ defined by $u \circ v=$ $\pi^{-1}(\pi(u) * \pi(v))(u, v \in L)$.

Lemma 8.4. $\quad L^{\prime}=(L, \circ)$ is a left quasigroup; the left division $\backslash_{L^{\prime}}$ of $L^{\prime}$ is defined by $u \backslash_{L^{\prime}} w=\pi^{-1}\left(\pi(u) \backslash_{G} \pi(w)\right)\left(u, w \in L^{\prime}\right)$.

Proof. The proof is straightforward, because $G$ is a left quasigroup and $\pi$ is a bijection.

By virtue of (3.1), (6.2), and the definition of the binary operation $\circ$,

$$
\begin{aligned}
& \xi_{\lambda}^{\left(L^{\prime},\left(G, \mu_{1}^{G}\right), \pi\right)}(u)(v) \\
= & \lambda \backslash_{L^{\prime}} \pi^{-1}\left(\mu_{1}^{G}(\pi(\lambda), \pi(\lambda \circ u), \pi((\lambda \circ u) \circ v))\right) \\
= & v
\end{aligned}
$$

for all $\lambda, u, v \in L^{\prime}$. With the aid of (3.2), (8.2), and Lemma 8.4,

$$
\eta_{\lambda}^{\left(L^{\prime},\left(G, \mu_{1}^{G}\right), \pi\right)}(v)(u)=\pi^{-1}\left((\pi(\lambda) * \pi(v)) \backslash_{G}((\pi(\lambda) * \pi(u)) * \pi(v))\right)
$$

for all $\lambda, u, v \in L^{\prime}$. This element $\eta_{\lambda}^{\left(L^{\prime},\left(G, \mu_{1}^{G}\right), \pi\right)}(v)(u)$ is independent of $\lambda$ because of (6.1). Hence, the dynamical YB map $R^{\left(L^{\prime},\left(G, \mu_{1}^{G}\right), \pi\right)}(\lambda)$ is a YB map.

From Proposition 8.1, the dynamical YB map $R(\lambda)=R^{\left(L,\left(G, \mu_{1}^{G}\right), \pi\right)}(\lambda)$ has a vertex-IRF correspondence whose vertex counterpart is this YB map $R^{\left(L^{\prime},\left(G, \mu_{1}^{G}\right), \pi\right)}(\lambda)$.

We can construct objects $V=(L, R(\lambda))$ of the category $\mathcal{D}_{1}$ such that each $R(\lambda)$ really depends on the dynamical parameter $\lambda$. Let $G=(G, *)$ be a quasigroup satisfying (6.1) (see Definition 2.2), and let $\circ$ denote the following binary operation on $G$ : $a \circ b=b(a, b \in G)$. Then $L^{\prime}=(G, \circ)$ is a left quasigroup, and (3.3) gives rise to that

$$
R^{\left(L^{\prime},\left(G, \mu_{1}^{G}\right), \operatorname{id}_{G}\right)}(\lambda)(u, v)=\left(v, \lambda *\left(u \backslash_{G} v\right)\right) \quad(\forall \lambda, u, v \in G) .
$$

On account of Propositions 6.9 and 6.14 , the pair $\left(L^{\prime}, R^{\left(L^{\prime},\left(G, \mu_{1}^{G}\right), \mathrm{id}_{G}\right)}(\lambda)\right)$ is an object of the category $\mathcal{D}_{1}$.

We suppose that $R^{\left(L^{\prime},\left(G, \mu_{1}^{G}\right), \operatorname{id}_{G}\right)}(\lambda)=R^{\left(L^{\prime},\left(G, \mu_{1}^{G}\right), \operatorname{id}_{G}\right)}\left(\lambda^{\prime}\right)$. Then $\lambda *$ $\left(u \bigwedge_{G} v\right)=\lambda^{\prime} *\left(u \backslash_{G} v\right)$ for all $u, v \in G$. This equation induces that $\lambda=\lambda^{\prime}$, because $G$ is a quasigroup. Hence, the dynamical YB map $R^{\left(L^{\prime},\left(G, \mu_{1}^{G}\right), \operatorname{id}_{G}\right)}(\lambda)$ is dependent on $\lambda$, unless $|G|=1$. 
Remark 8.5. Let $L$ and $G$ be groups, and $\pi$ a bijective 1-cocycle of the group $L$ with coefficients in the group $G$. From Remarks 2.6 and 6.7, $R^{\left(L,\left(G, \mu_{1}^{G}\right), \pi\right)}(\lambda)$ is the YB map in [10]. Let us suppose that $|L| \neq 1$ (hence $|G| \neq 1)$. The YB map $R^{\left(L,\left(G, \mu_{1}^{G}\right), \pi\right)}(\lambda)$ has a vertex-IRF correspondence whose IRF counterpart is the dynamical YB map $R^{\left(L^{\prime},\left(G, \mu_{1}^{G}\right), \operatorname{id}_{G}\right)}(\lambda)\left(\lambda \in L^{\prime}(=G)\right)$ (8.3).

\section{References}

[1] V. M. Bukhshtaber, The Yang-Baxter transformation, Uspekhi Mat. Nauk 53 (1998), no. 6(324), 241-242; translation in Russian Math. Surveys 53 (1998), no. 6, 1343-1345.

[2] V. G. Drinfel'd, On some unsolved problems in quantum group theory, in Quantum groups (Leningrad, 1990), 1-8, Lecture Notes in Math., 1510, Springer, Berlin.

[3] P. Etingof, Geometric crystals and set-theoretical solutions to the quantum Yang-Baxter equation, Comm. Algebra 31 (2003), no. 4, 1961-1973.

[4] P. Etingof and F. Latour, The dynamical Yang-Baxter equation, representation theory, and quantum integrable systems, Oxford Univ. Press, Oxford, 2005.

[5] P. Etingof, T. Schedler and A. Soloviev, Set-theoretical solutions to the quantum YangBaxter equation, Duke Math. J. 100 (1999), no. 2, 169-209.

[6] T. Gateva-Ivanova, A combinatorial approach to the set-theoretic solutions of the YangBaxter equation, J. Math. Phys. 45 (2004), no. 10, 3828-3858.

[7] G. Hatayama, A. Kuniba, M. Okado, T. Takagi and Y. Yamada, Scattering rules in soliton cellular automata associated with crystal bases, in Recent developments in infinitedimensional Lie algebras and conformal field theory (Charlottesville, VA, 2000), 151182, Contemp. Math., 297, Amer. Math. Soc., Providence, RI.

[8] C. Kassel, Quantum groups, Springer, New York, 1995.

[9] T. H. Koornwinder, Some details of proofs of theorems related to the quantum dynamical Yang-Baxter equation, Int. J. Math. Math. Sci. 24 (2000), no. 12, 793-806.

[10] J.-H. Lu, M. Yan and Y.-C. Zhu, On the set-theoretical Yang-Baxter equation, Duke Math. J. 104 (2000), no. 1, 1-18.

[11] S. Mac Lane, Categories for the working mathematician, Second edition, Springer, New York, 1998.

[12] H. O. Pflugfelder, Quasigroups and loops: introduction, Heldermann, Berlin, 1990.

[13] Y. Shibukawa, Dynamical Yang-Baxter maps, Int. Math. Res. Not. 2005 (2005), no. 36, 2199-2221.

[14] J. D. H. Smith and A. B. Romanowska, Post-modern algebra, Wiley, New York, 1999.

[15] A. P. Veselov, Yang-Baxter maps and integrable dynamics, Phys. Lett. A 314 (2003), no. 3, 214-221.

[16] A. Weinstein and P. Xu, Classical solutions of the quantum Yang-Baxter equation, Comm. Math. Phys. 148 (1992), no. 2, 309-343. 